\title{
ON THE LOCATION OF THE PEAKS OF LEAST-ENERGY SOLUTIONS TO SEMILINEAR DIRICHLET PROBLEMS WITH CRITICAL GROWTH
}

\author{
MARCO A. S. SOUTO
}

Received 20 December 2001

We study the location of the peaks of solution for the critical growth problem $-\varepsilon^{2} \Delta u+u=f(u)+u^{2^{*}-1}, u>0$ in $\Omega, u=0$ on $\partial \Omega$, where $\Omega$ is a bounded domain; $2^{*}=2 N /(N-2), N \geq 3$, is the critical Sobolev exponent and $f$ has a behavior like $u^{p}, 1<p<2^{*}-1$.

\section{Introduction}

In this paper, we will study the location of the peaks of least-energy solution for the problem

$$
\begin{aligned}
-\varepsilon^{2} \Delta u+u & =f(u)+u^{2^{*}-1} \quad \text { in } \Omega \\
u>0 & \text { in } \Omega \\
u=0 & \text { on } \partial \Omega
\end{aligned}
$$

where $\Omega$ is a bounded domain in $\mathbb{R}^{N}, \varepsilon>0$, and $f$ is a function satisfying some subcritical conditions. Here $2^{*}=2 N /(N-2), N \geq 3$, is the critical Sobolev exponent.

By least-energy solution for problem (1.1) we mean a critical point at the Mountain-Pass level of the associated energy functional

$$
J_{\mathcal{\varepsilon}}(u)=\frac{1}{2} \int_{\Omega}\left(\varepsilon^{2}|\nabla u|^{2}+u^{2}\right) d z-\int_{\Omega}\left[F(u)+\frac{1}{2^{*}}\left(u^{+}\right)^{2^{*}}\right] d z,
$$

(where $u^{+}=\max \{u, 0\}$ ), defined on the Hilbert space $H_{o}^{1}(\Omega)$ endowed with the norm

$$
\|u\|_{\varepsilon}^{2}=\int_{\Omega}\left(\varepsilon^{2}|\nabla u|^{2}+u^{2}\right) d z
$$


The Mountain-Pass level of $J_{\varepsilon}$ is defined by

$$
c_{\varepsilon}=\inf _{g \in \Gamma} \max _{0 \leq t \leq 1} J_{\mathcal{\varepsilon}}(g(t))
$$

where $\Gamma$ is the set of all continuous paths joining the origin and a fixed nonzero element $e$ in $H_{o}^{1}(\Omega)$, such that $e \neq 0$ and $J_{\varepsilon}(e) \leq 0$. Under suitable hypothesis (e.g., $\left(\mathrm{f}_{1}\right),\left(\mathrm{f}_{4}\right),\left(\mathrm{f}_{5}\right)$ below), it is not hard to check that $c_{\varepsilon}>0$ does not depend on the element $0 \neq v \in H_{o}^{1}(\Omega)$ and $u$ is a least-energy solution if and only if $J_{\varepsilon}(u)=c$ and $J_{\varepsilon}^{\prime}(u)=0$, and $J_{\mathcal{\varepsilon}}(u) \leq J_{\mathcal{\varepsilon}}(v)$ for all $v \neq 0$ such that $J_{\mathcal{\varepsilon}}^{\prime}(v)=0$.

The existence of least-energy solution of problem (1.1) was given in Brézis and Nirenberg in [3, Theorem 2.1] (see Lemma 2.4 in this paper).

In this paper, we will study some properties of the least-energy solution $u_{\varepsilon}$ of problem (1.1) when $\varepsilon$ is small. In order to describe these properties, we introduce the hypotheses on the function $f$.

Suppose that $f: \mathbb{R}_{+} \rightarrow \mathbb{R}_{+}$is a $C^{1, \alpha}$ function such that

$\left(\mathrm{f}_{1}\right) f(0)=f^{\prime}(0)=0$;

$\left(\mathrm{f}_{2}\right)$ there is $q_{1} \in(1,(N+2) /(N-2))$ such that

$$
\lim _{s \rightarrow \infty} \frac{f(s)}{s^{q_{1}}}=0
$$

$\left(\mathrm{f}_{3}\right)$ there are $q_{2} \in(1,(N+2) /(N-2))$ and $\lambda>0$ such that

$$
f(s) \geq \lambda s^{q_{2}}, \quad \forall s>0
$$

(when $N=3$, we need $q_{2}>2$, otherwise we require a sufficiently large $\lambda$ ); $\left(\mathrm{f}_{4}\right)$ if $F(s)=\int_{o}^{s} f(t) d t$, for some $\theta \in\left(2, q_{1}+1\right)$ we have

$$
0<\theta F(s) \leq f(s) s, \quad \forall s>0
$$

$\left(\mathrm{f}_{5}\right)$ the function $f(s) / s$ is increasing for $s>0$.

Since our interest is on positive solutions we define $f(s)=0$, in $s \leq 0$. Now we will state our main result.

Theorem 1.1. Suppose that $\Omega$ is a bounded domain in $\mathbb{R}^{N} ; f$ satisfies $\left(\mathrm{f}_{1}\right),\left(\mathrm{f}_{2}\right)$, $\left(f_{3}\right),\left(f_{4}\right),\left(f_{5}\right)$; and let $u_{\varepsilon}$ be the least-energy solution of (1.1). Then, there is a $\varepsilon_{o}>0$ such that

(i) $u_{\varepsilon}$ attains only one local maximum at some $z_{\varepsilon} \in \Omega$ (hence global maximum), for all $\varepsilon \in\left(0, \varepsilon_{o}\right]$;

(ii) $u_{\varepsilon}$ converges uniformly to zero over compact subsets of $\Omega \backslash\left\{z_{\varepsilon}\right\}$ as $\varepsilon \rightarrow 0$;

(iii) $\operatorname{dist}\left(z_{\varepsilon}, \partial \Omega\right) \rightarrow \max _{z \in \Omega} \operatorname{dist}(z, \partial \Omega)$. 
This statement is analogous to the one given by $\mathrm{Ni}$ and Wei in [8], in the subcritical case

$$
\begin{gathered}
-\varepsilon^{2} \Delta u+u=h(u), \quad \text { in } \Omega, \\
u=0, \quad \text { on } \partial \Omega,
\end{gathered}
$$

where $h$ satisfies the following hypothesis:

(i) $\left(f_{1}\right),\left(f_{2}\right),\left(f_{4}\right)$, and $\left(f_{5}\right)$ hold;

(ii) the global problem

$$
-\Delta u+u=h(u), \quad \text { in } \mathbb{R}^{N}
$$

has a unique positive solution in $H^{1}\left(\mathbb{R}^{N}\right)$;

(iii) this solution is nondegenerate in the sense that

$$
-\Delta v+v=h^{\prime}(u) v, \quad \text { in } \mathbb{R}^{N}
$$

has no nontrivial spherically symmetric solution in $L^{2}\left(\mathbb{R}^{N}\right)$.

In [8], Ni and Wei also have described the asymptotic profile (in $\varepsilon$ ) of $u_{\varepsilon}$, giving a detailed description for $\varepsilon$ small. Here in the critical case, the solutions have the same profile.

In this work we will show that a ground state solution of the critical problem (1.1) is also solution of a subcritical problem (1.8) by showing that for small $\varepsilon$ we have a uniform bound for the $L^{\infty}$ norm of $u_{\varepsilon}$.

The difficulty here lies in finding an upper bound for $\left\|u_{\varepsilon}\right\|_{L^{\infty}(\Omega)}$ by obtaining a bound for $u_{\varepsilon}$ in $L^{p}(\Omega)$ norm, for all $p \geq 2$. In the subcritical case this boundedness is obtained since the family $u_{\varepsilon}$ is bounded in $H^{1}(\Omega)$ but this argument does not work in the critical case. Here, we obtain an $L^{\infty}$-bound for $u_{\varepsilon}$ through the estimate below, which is based on Moser's iteration technique (see [11]) and is essentially due to Brézis and Kato [2].

Proposition 1.2. Let $\Lambda$ be an open subset and $q \in L^{N / 2}(\Lambda)$. Suppose that $g: \Lambda \times$ $\mathbb{R} \rightarrow \mathbb{R}$ is a Caratheodory function satisfying

$$
|g(x, s)| \leq\left(q(x)+C_{g}\right)|s|, \quad \forall s \in \mathbb{R}, x \in \Lambda \text { and for some } C_{g}>0 .
$$

Then, if $v \in H_{o}^{1}(\Lambda)$ is such that

$$
-\Delta v=g(x, v), \quad \text { in } \Lambda
$$

we have $v \in L^{p}(\Lambda)$ for all $2 \leq p<\infty$. Moreover, there is a positive constant $C_{p}=$ $C\left(p, C_{g}, q\right)$ such that

$$
\|v\|_{L^{2^{*}(p+1)}(\Lambda)} \leq C_{p}\|v\|_{L^{2(p+1)}(\Lambda)} .
$$

Remark 1.3. The dependence on $q$ of $C_{p}$ can be given uniformly on a family of functions $\left\{q_{\varepsilon}\right\}_{\varepsilon>0}$ such that $q_{\varepsilon}$ converges in $L^{N / 2}$ (see the appendix). 
We have organized this paper as follows: the next section contains the proof of Theorem 1.1. This proof consists in a series of lemmas which show the $L^{\infty}$ bound for $u_{\varepsilon}$, where these functions are solutions of a class of subcritical problems (1.8). The third section is an appendix proving Proposition 1.2, for the sake of completeness.

\section{Proof of Theorem 1.1}

Before proving Theorem 1.1, let us fix some notation and preliminaries.

Remark 2.1. Throughout this section, we use the equivalent characterization of $c_{\varepsilon}$, which is more adequate to our purposes, given by

$$
\mathcal{c}_{\varepsilon}=\inf _{v \in H_{o}^{1}(\Omega) \backslash\{0\}} \max _{t \geq 0} J_{\varepsilon}(t v) .
$$

(see Willem [13, Theorem 4.2]).

We denote by $J: H^{1}\left(\mathbb{R}^{N}\right) \rightarrow \mathbb{R}$ the functional given by

$$
J(u)=\frac{1}{2}\|u\|^{2}-\int_{\mathbb{R}^{N}}\left[F(u)+\frac{1}{2^{*}}\left(u_{+}\right)^{2^{*}}\right] d x,
$$

where

$$
\|u\|^{2}=\int_{\mathbb{R}^{N}}\left(|\nabla u|^{2}+u^{2}\right) d x
$$

associated with the problem

$$
-\Delta u+u=f(u)+|u|^{2^{*}-2} u, \quad \text { in } \mathbb{R}^{N} .
$$

It is known that under assumptions $\left(f_{1}\right),\left(f_{2}\right),\left(f_{3}\right),\left(f_{4}\right),\left(f_{5}\right)$, and $(2.4)$ possesses a ground state solution $\omega$ in the level

$$
c=J(\omega)=\inf _{v \in H^{1}\left(\mathbb{R}^{N}\right) \backslash\{0\}} \max _{t \geq 0} J(t v),
$$

(see [1]).

Remark 2.2. It is easy to check that for each nonzero $v$ in $H^{1}\left(\mathbb{R}^{N}\right)$, there is a unique $t_{o}=t(v)$ such that

$$
J\left(t_{o} v\right)=\max _{t \geq 0} J(t v)
$$

Indeed, since

$$
J(t v)=\frac{t^{2}}{2}\|v\|^{2}-\int_{\mathbb{R}^{N}}\left[F(t v)-\frac{t^{2^{*}}}{2^{*}}\left(v^{+}\right)^{2^{*}}\right] d x, \quad \text { for } t \geq 0,
$$


the maximum point $t_{o}$ of $J(t v)$ is given by

$$
\|v\|^{2}=\int_{\mathbb{R}^{N}}\left[t_{o}^{-1} v f\left(t_{o} v\right)+t_{o}^{2^{*}-2}\left(v^{+}\right)^{2^{*}}\right] d x .
$$

We assume, without loss of generality that $0 \in \Omega$. Set $\Omega_{\varepsilon}=\left\{x \in \mathbb{R}^{N} ; \varepsilon x \in \Omega\right\}$.

The restriction of $J$ to $H_{o}^{1}\left(\Omega_{\varepsilon}\right)$ is the energy functional,

$$
J(u)=\frac{1}{2} \int_{\Omega_{\varepsilon}}\left(|\nabla u|^{2}+u^{2}\right) d x-\int_{\Omega_{\varepsilon}}\left[F\left(u_{+}\right)+\frac{1}{2^{*}} u_{+}^{2^{*}}\right] d x, \quad u \in H_{o}^{1}\left(\Omega_{\varepsilon}\right),
$$

associated with the problem

$$
\begin{aligned}
-\Delta u+u & =f(u)+u^{2^{*}-1} \quad \text { in } \Omega_{\varepsilon} \\
u & =0 \quad \text { on } \partial \Omega_{\varepsilon} .
\end{aligned}
$$

If $u_{\varepsilon}$ is a critical point of $J_{\varepsilon}$, the family

$$
v_{\varepsilon}(x)=u_{\varepsilon}(z)=u_{\varepsilon}(\varepsilon x), \quad z=\varepsilon x
$$

is such that each $v_{\varepsilon}$ is a critical point of functional $J$ restricted to $H_{o}^{1}\left(\Omega_{\varepsilon}\right)$ at the level

$$
b_{\varepsilon}=J\left(v_{\varepsilon}\right)=\inf _{v \in H_{o}^{1}\left(\Omega_{\varepsilon}\right) \backslash\{0\}} \max _{t \geq 0} J(t v) .
$$

It is easy to check that $b_{\varepsilon}=\varepsilon^{-N} c_{\varepsilon}$ and from the definition of $c$ it follows that $b_{\varepsilon} \geq c$ for all $\varepsilon>0$.

We will start with the following property of $\left\{b_{\varepsilon}\right\}_{\varepsilon>0}$.

LEMMA 2.3. For $\left\{b_{\varepsilon}\right\}_{\varepsilon>0}, \lim _{\varepsilon \rightarrow 0} b_{\varepsilon}=c$.

Proof. Fix $\omega$ a ground state solution of problem (2.4) and let $\psi_{\varepsilon}(x)=\varphi(\varepsilon x) \omega(x)$, where $\varphi$ is a $C^{1}$-function such that

$$
\varphi(x)= \begin{cases}1 & \text { if } x \in B_{1} \\ 0 & \text { if } x \notin B_{2}\end{cases}
$$

$B_{1}=B_{\rho}(0), B_{2}=B_{2 \rho}(0) \subset \Omega$. Observe that $\psi_{\varepsilon} \rightarrow \omega$ in $H^{1}\left(\mathbb{R}^{N}\right)$ and the support of $\psi_{\varepsilon}$ is in $\Omega_{\varepsilon}$. By definition of $b_{\varepsilon}$, we have $t_{\varepsilon}>0$ such that

$$
b_{\varepsilon} \leq \max _{t>0} J\left(t \psi_{\varepsilon}\right)=J\left(t_{\varepsilon} \psi_{\varepsilon}\right)
$$


552 Location of the peaks of solutions to critical growth problems

From (2.8) and condition $\left(\mathrm{f}_{3}\right)$ it follows that

$$
\begin{aligned}
\left\|\psi_{\varepsilon}\right\|^{2} & =\int_{\mathbb{R}^{N}}\left[t_{\varepsilon}^{-1} \psi_{\varepsilon} f\left(t_{\varepsilon} \psi_{\varepsilon}\right)+t_{\varepsilon}^{2^{*}-2} \psi_{\varepsilon}^{2^{*}}\right] d x \\
& \geq \int_{\mathbb{R}^{N}}\left[\lambda t_{\varepsilon}^{q_{2}-1} \psi_{\varepsilon}^{q_{2}+1}+t_{\varepsilon}^{2^{*}-2} \psi_{\varepsilon}^{2^{*}}\right] d x,
\end{aligned}
$$

so that, $t_{\varepsilon}$ is bounded. Equality (2.15) and Remark 2.2 show that $t_{\varepsilon} \rightarrow t(\omega)=1$, as $\varepsilon \rightarrow 0$. Then we have $t_{\varepsilon} \psi_{\varepsilon} \rightarrow \omega$ in $H^{1}\left(\mathbb{R}^{N}\right)$ and

$$
\lim _{\varepsilon \rightarrow 0} J\left(t_{\varepsilon} \psi_{\varepsilon}\right)=J(\omega)=c
$$

Combining (2.14), (2.16), and the inequality $b_{\varepsilon} \geq c$, for all $\varepsilon>0$, we have proved this lemma.

Lemma 2.4. The inequality $c<(1 / N) S^{N / 2}$ holds, where $S$ is the best Sobolev constant for the embedding $D^{1,2}\left(\mathbb{R}^{N}\right) \hookrightarrow L^{2^{*}}\left(\mathbb{R}^{N}\right)$.

Proof. For each $h>0$, consider the function

$$
\phi_{h}(x)=\frac{[N(N-2) h]^{(N-2) / 4}}{\left(h+|x|^{2}\right)^{(N-2) / 2}} .
$$

We recall that $\phi_{h}$ satisfies the problem

$$
\begin{gathered}
-\Delta u=u^{2^{*}-1} \quad \text { in } \mathbb{R}^{N}, \\
u(x)>0, \quad \int_{\mathbb{R}^{N}}|\nabla u|^{2} d x<\infty, \\
\int_{\mathbb{R}^{N}}\left|\nabla \phi_{h}\right|^{2} d x=\int_{\mathbb{R}^{N}} \phi_{h}^{2^{*}} d x=S^{N / 2} \quad \text { (see Talenti [12]). }
\end{gathered}
$$

Now, consider $\psi_{h}(x)=\varphi \phi_{h}(x) /\left\|\varphi \phi_{h}\right\|_{L^{2^{*}}\left(\mathbb{R}^{N}\right)}$, where $\varphi$ is the function defined in the proof of Lemma 2.3. From condition $\left(f_{3}\right)$ we have

$$
J\left(t \psi_{h}\right) \leq \frac{t^{2}}{2} \int_{B_{2}}\left(\left|\nabla \psi_{h}\right|^{2}+\psi_{h}^{2}\right) d x-\frac{\lambda t^{q_{2}+1}}{q_{2}+1} \int_{B_{2}} \psi_{h}^{q_{2}+1} d x-\frac{t^{2^{*}}}{2^{*}} .
$$

Using arguments as in [7], there exists $h>0$ such that

$$
\max _{t \geq 0}\left\{\frac{t^{2}}{2} \int_{B_{2}}\left(\left|\nabla \psi_{h}\right|^{2}+\psi_{h}^{2}\right) d x-\frac{\lambda t^{q_{2}+1}}{q_{2}+1} \int_{B_{2}} \psi_{h}^{q_{2}+1} d x-\frac{t^{2^{*}}}{2^{*}}\right\}<\frac{1}{N} S^{N / 2} .
$$


Therefore, from (2.19) and (2.20) we have that

$$
\max _{t \geq 0} J\left(t \psi_{h}\right)<\frac{1}{N} S^{N / 2},
$$

and the proof of the lemma is completed.

Notice that the same proof of Lemma 2.4 can be used to show that $b_{\varepsilon}<$ $(1 / N) S^{N / 2}$, for all $\varepsilon>0$. Using [3, Theorem 2.1], this inequality implies the existence of $v_{\varepsilon}$ and then the existence of $u_{\varepsilon}$.

Lemma 2.5. There are $\varepsilon_{o}>0$; a family $\left\{y_{\varepsilon}\right\}_{\left\{0<\varepsilon \leq \varepsilon_{0}\right\}} \subset \mathbb{R}^{N}, y_{\varepsilon} \in \Omega_{\varepsilon}$; constants $R>0$ and $\beta>0$ such that

$$
\begin{gathered}
\int_{B_{R}\left(y_{\varepsilon}\right)} v_{\varepsilon}^{2} d x \geq \beta>0, \quad \forall 0<\varepsilon \leq \varepsilon_{o}, \\
\lim _{\varepsilon \rightarrow o} d\left(y_{\varepsilon}, \partial \Omega_{\varepsilon}\right)=\infty .
\end{gathered}
$$

Proof. Start by showing that there is a family satisfying inequality (2.22). Arguing to the contrary, there is $\varepsilon_{n} \backslash 0$ such that for all $R>0$

$$
\lim _{n \rightarrow \infty} \sup _{x \in \mathbb{R}^{N}} \int_{B_{R}(x)} v_{\varepsilon_{n}}^{2} d x=0 .
$$

Using (Lions [6, Lemma I.1]) we have

$$
\int_{\mathbb{R}^{N}} v_{\mathcal{E}_{n}}^{q} d x=o_{n}(1), \quad \text { as } n \longrightarrow \infty, \forall 2<q<2^{*},
$$

and, from $\left(f_{1}\right)$ and $\left(f_{2}\right)$,

$$
\int_{\mathbb{R}^{N}} F\left(v_{\varepsilon_{n}}\right) d x=\int_{\mathbb{R}^{N}} v_{\varepsilon_{n}} f\left(v_{\varepsilon_{n}}\right) d x=o_{n}(1) .
$$

Since $J^{\prime}\left(v_{\varepsilon_{n}}\right) \cdot v_{\varepsilon_{n}}=0$, we conclude from (2.26) that

$$
\left\|v_{\varepsilon_{n}}\right\|^{2}=\int_{\mathbb{R}^{N}} v_{\varepsilon_{n}}^{2^{*}} d x+o_{n}(1)
$$

Let $\ell \geq 0$ be such that $\left\|v_{\varepsilon_{n}}\right\|^{2} \rightarrow \ell$. Passing to the limit in $J\left(v_{\varepsilon_{n}}\right)=b_{\varepsilon_{n}}$ and using (2.26) we have

$$
\ell=N c
$$

and hence $\ell>0$. Now, using the definition of the constant $S$, we have

$$
\left\|v_{\varepsilon_{n}}\right\|^{2} \geq S\left(\int_{\mathbb{R}^{N}} v_{\varepsilon_{n}}^{2^{*}} d x\right)^{2 / 2^{*}} .
$$


Taking the limit in the above inequalities, as $n \rightarrow \infty$, we achieve that

$$
\ell \geq S \ell^{2 / 2^{*}}
$$

and by (2.28), that

$$
c \geq \frac{1}{N} S^{N / 2}
$$

which contradicts Lemma 2.4 and then (2.22) holds.

Finally, to establish (2.23), suppose the contrary. That is, there exist $\varepsilon_{n} \rightarrow 0$ and $R>0$ such that $\operatorname{dist}\left(y_{\varepsilon_{n}}, \partial \Omega_{\varepsilon_{n}}\right) \leq R$, hence $\operatorname{dist}\left(\varepsilon_{n} y_{\varepsilon_{n}}, \partial \Omega\right) \leq \varepsilon_{n} R$. Without loss of generality, we have $\varepsilon_{n} y_{\varepsilon_{n}} \rightarrow y_{o}$ for some $y_{o} \in \partial \Omega$. The arguments that follow can be found in [8].

Let $v$ be the unit interior normal to $\partial \Omega$ at $y_{o}$, and $\delta>0$ such that $B_{\delta}\left(y_{o}+\right.$ $\delta v) \subset \Omega$ and $B_{\delta}\left(y_{o}-\delta v\right) \cap \Omega=\varnothing$. Let $\Omega_{n}=\left\{x \in \mathbb{R}^{N}: y_{o}+\varepsilon_{n} x \in \Omega\right\}$ and $w_{n}(x)$ $=u_{\varepsilon_{n}}\left(y_{o}+\varepsilon_{n} x\right)$. This sequence $w_{n}$ is bounded in $H^{1}\left(\mathbb{R}^{N}\right),-\Delta w_{n}+w_{n}=f\left(w_{n}\right)+$ $w_{n}^{2^{*}-1}$ in $\Omega_{n}$,

$$
\int_{B_{2 R}(0)} w_{n}^{2} d x \geq \int_{B_{R}\left(y_{\varepsilon_{n}}\right)} v_{\varepsilon_{n}}^{2} d x \geq \beta>0, \quad \forall n,
$$

and we have that $w_{n}$ converges weakly to some $w$ in $H^{1}\left(\mathbb{R}^{N}\right)$.

Let $\mathbb{R}_{+, v}^{N}$ be the half space $\left\{x \in \mathbb{R}^{N}: x \cdot v>0\right\}$. Notice that $B_{\varepsilon_{n}^{-1} \delta}\left(\varepsilon_{n}^{-1} \delta v\right) \subset \Omega_{n}$ and $B_{\varepsilon_{n}^{-1} \delta}\left(-\varepsilon_{n}^{-1} \delta v\right) \cap \Omega_{n}=\varnothing$ and then we can prove that for all compacts $K_{+} \subset$ $\mathbb{R}_{+, v}^{N}$ and $K_{-} \subset \mathbb{R}_{-, v}^{N}=\mathbb{R}^{N} \backslash \overline{\mathbb{R}_{+, v}^{N}}$, we have $K_{+} \subset \Omega_{n}$ and $K_{-} \cap \Omega_{n}=\varnothing$, for $n$ large.

Then for each $\phi \in C_{o}^{\infty}\left(\mathbb{R}_{+, v}^{N}\right)$ such that $\operatorname{supp} \phi \subset \Omega_{n}$, we have

$$
\int_{\mathbb{R}_{+, v}^{N}}\left(\nabla w_{n} \nabla \phi+w_{n} \phi\right) d x=\int_{\mathbb{R}_{+, v}^{N}}\left(f\left(w_{n}\right)+w_{n}^{2^{*}-1}\right) \phi d x
$$

From (2.33), usual arguments show that $w \in H^{1}\left(\mathbb{R}^{N}\right) \cap C^{2}\left(\mathbb{R}_{+}^{N}\right)$ and satisfies $-\Delta w+w=f(w)+w^{2^{*}-1}$, in $\mathbb{R}_{+, v}^{N}$, and $w \equiv 0$ in $\mathbb{R}_{-, v}^{N}$. Theorem I.1, due to Esteban and Lions in [4], shows that $w \equiv 0$ which contradicts

$$
\int_{B_{2 R}(0) \cap \mathbb{R}_{+, v}^{N}} w^{2} d x \geq \beta>0 .
$$

This completes the proof of the lemma.

Now we will consider the translation of $v_{\varepsilon}$, defined by $\omega_{\mathcal{\varepsilon}}(x)=v_{\varepsilon}\left(x+y_{\varepsilon}\right)=$ $u_{\varepsilon}\left(\varepsilon y_{\varepsilon}+\varepsilon x\right)$ in $\widetilde{\Omega}_{\varepsilon}=\left\{x \in \mathbb{R}^{N} ; \varepsilon y_{\varepsilon}+\varepsilon x \in \Omega\right\}$ and $\omega_{\varepsilon}=0$ outside $\widetilde{\Omega}_{\varepsilon}$. From (2.23), any compact subset of $\mathbb{R}^{N}$ is contained in $\widetilde{\Omega}_{\varepsilon}$, for $\varepsilon$ sufficiently small.

From Lemma 2.5,

$$
\int_{B_{R}(0)} \omega_{\varepsilon}^{2} d x \geq \beta>0, \quad \forall 0<\varepsilon \leq \varepsilon_{o} .
$$


Consider a sequence $\varepsilon_{n} \succ 0$ and set $\widetilde{\Omega}_{n}=\widetilde{\Omega}_{\varepsilon_{n}}, \omega_{n}=\omega_{\varepsilon_{n}}, v_{n}=v_{\varepsilon_{n}}, y_{\varepsilon}=y_{\varepsilon_{n}}$.

We will prove that $\omega_{n}$ is bounded in the $L^{\infty}$ norm. In that case, $u_{\varepsilon}$ is also bounded in $L^{\infty}(\Omega)$ norm and the proof of Theorem 1.1 follows from the subcritical case, as Lemma 2.8 will show.

Since the sequence $\omega_{n}$ a translation of $v_{n}$, we have a uniform bound for $\left\|\omega_{n}\right\|$ and there is a $\omega_{o} \in H^{1}\left(\mathbb{R}^{N}\right)$ which is weak limit of $\omega_{n}$ in $H^{1}\left(\mathbb{R}^{N}\right)$. From (2.35) we have $\omega_{o} \neq 0$. We can write limit (2.23) in the following form

$$
\lim _{n \rightarrow \infty} d\left(0, \partial \widetilde{\Omega}_{n}\right)=\infty
$$

Then for each $\phi \in C_{o}^{\infty}\left(\mathbb{R}^{N}\right)$ and large $n$ such that supp $\phi \subset \widetilde{\Omega}_{n}$, we have

$$
\int_{\mathbb{R}^{N}}\left(\nabla \omega_{n} \nabla \phi+\omega_{n} \phi\right) d x=\int_{\mathbb{R}^{N}}\left(f\left(\omega_{n}\right)+\omega_{n}^{2^{*}-1}\right) \phi d x, \quad \forall n .
$$

From (2.37), usual arguments show that $\omega_{o}$ is a solution of problem (2.4), hence a critical point of $J$, and $J\left(\omega_{o}\right) \geq c$.

Lemma 2.6. The sequence $\omega_{n}$ converges to $\omega_{o}$ in $H^{1}\left(\mathbb{R}^{N}\right)$ and $J\left(\omega_{o}\right)=c$.

Proof. This fact comes from Lemma 2.5 and Fatou's lemma applied in the positive sequence $\omega_{n} f\left(\omega_{n}\right)-\theta F\left(\omega_{n}\right)$. Observe that

$$
\begin{aligned}
b_{\varepsilon_{n}} & =J\left(v_{n}\right)-\frac{1}{\theta} J^{\prime}\left(v_{n}\right) v_{n} \\
& =\left(\frac{\theta-2}{2 \theta}\right)\left\|v_{n}\right\|^{2}+\frac{1}{\theta} \int_{\mathbb{R}^{N}}\left[v_{n} f\left(v_{n}\right)-\theta F\left(v_{n}\right)\right]+\left(\frac{2^{*}-\theta}{2 * \theta}\right) \int_{\mathbb{R}^{N}} v_{n}^{2^{*}} \\
& =\left(\frac{\theta-2}{2 \theta}\right)\left\|\omega_{n}\right\|^{2}+\frac{1}{\theta} \int_{\mathbb{R}^{N}}\left[\omega_{n} f\left(\omega_{n}\right)-\theta F\left(\omega_{n}\right)\right]+\left(\frac{2^{*}-\theta}{2 * \theta}\right) \int_{\mathbb{R}^{N}} \omega_{n}^{2^{*}} .
\end{aligned}
$$

From (2.38)

$$
\begin{aligned}
c & \leq J\left(\omega_{o}\right)=J\left(\omega_{o}\right)-\frac{1}{\theta} J^{\prime}\left(\omega_{o}\right) \omega_{o} \\
& =\left(\frac{\theta-2}{2 \theta}\right)\left\|\omega_{o}\right\|^{2}+\frac{1}{\theta} \int_{\mathbb{R}^{N}}\left[\omega_{o} f\left(\omega_{o}\right)-\theta F\left(\omega_{o}\right)\right]+\left(\frac{2^{*}-\theta}{2 * \theta}\right) \int_{\mathbb{R}^{N}} \omega_{o}^{2^{*}} \\
& \leq \liminf \left(\frac{\theta-2}{2 \theta}\right)\left\|\omega_{n}\right\|^{2}+\frac{1}{\theta} \int_{\mathbb{R}^{N}}\left[\omega_{n} f\left(\omega_{n}\right)-\theta F\left(\omega_{n}\right)\right]+\left(\frac{2^{*}-\theta}{2^{*} \theta}\right) \int_{\mathbb{R}^{N}} \omega_{n}^{2^{*}} \\
& =\lim _{n \rightarrow \infty} b_{\mathcal{\varepsilon}_{n}}=c .
\end{aligned}
$$

We have proved that $J\left(\omega_{0}\right)=c$ and then (2.39) becomes an equality. 
556 Location of the peaks of solutions to critical growth problems

Combining (2.39) with the three following inequalities:

$$
\begin{aligned}
\left\|\omega_{o}\right\|^{2} & \leq \liminf \left\|\omega_{n}\right\|^{2}, \\
\int_{\mathbb{R}^{N}}\left[\omega_{o} f\left(\omega_{o}\right)-\theta F\left(\omega_{o}\right)\right] d x & \leq \liminf \int_{\mathbb{R}^{N}}\left[\omega_{n} f\left(\omega_{n}\right)-\theta F\left(\omega_{n}\right)\right] d x, \\
\int_{\mathbb{R}^{N}} \omega_{o}^{2^{*}} d x & \leq \liminf \int_{\mathbb{R}^{N}} \omega_{n}^{2^{*}} d x,
\end{aligned}
$$

we conclude that $\left\|\omega_{n}\right\| \rightarrow\left\|\omega_{o}\right\|$ and then $\omega_{n} \rightarrow \omega_{o}$ in $H^{1}\left(\mathbb{R}^{N}\right)$.

We are ready to conclude the proof of our main result. From Proposition 1.2 and Remark 1.3 with $q(x)=\omega_{n}^{2^{*}-2} \in L^{N / 2} ; g(x, s)=f(s)+s^{2^{*}}-s$, we have $\omega_{n} \in$ $L^{t}$ for all $t \geq 2$ and

$$
\left\|\omega_{n}\right\|_{L^{t}} \leq C_{t}
$$

where $C_{t}$ does not depend on $n$.

Now we will make use of a very particular version of [5, Theorem 8.17], due to Trudinger.

Proposition 2.7. Suppose that $t>N, g \in L^{t / 2}(\Lambda)$, and $u \in H_{o}^{1}(\Lambda)$ satisfies (in the weak sense)

$$
-\Delta u+u \leq \tilde{g}(x)
$$

where $\Lambda$ is an open subset of $\mathbb{R}^{N}$. Then for any ball $B_{2 R}(y) \subset \Lambda$,

$$
\sup _{B_{R}(y)} u \leq C\left(\left\|u^{+}\right\|_{L^{2}\left(B_{2 R}(y)\right)}+\|g\|_{L^{t / 2}\left(B_{2 R}(y)\right)}\right),
$$

where $C$ depends on $N$, $t$, and $R$.

We know that each $\omega_{n}$ satisfies

$$
-\Delta \omega_{n}+\omega_{n}=\omega_{n}^{2^{*}-1}+f\left(\omega_{n}\right), \quad \text { in } \widetilde{\Omega}_{n}
$$

and this implies that

$$
-\Delta \omega_{n}+\omega_{n} \leq g_{n}(x)=\omega_{n}^{2^{*}-1}+f\left(\omega_{n}\right), \quad \text { in } \mathbb{R}^{N}
$$

in the weak sense.

Since (2.41) holds, $\left\|g_{n}\right\|_{L^{t}}$ is bounded from above for some $t>N$. Using Proposition 2.7 in (2.45) we have

$$
\sup _{B_{1}(y)} \omega_{n} \leq C\left(\left\|\omega_{n}\right\|_{L^{2}\left(B_{2 R}(y)\right)}+\left\|g_{n}\right\|_{L^{t}\left(B_{2 R}(y)\right)}\right)
$$


for all $y \in \mathbb{R}^{N}$, which implies that there is a constant $a>0$, independent of $n$, such that

$$
\omega_{n}(x) \leq a, \quad \forall x \in \mathbb{R}^{N}
$$

It follows that there is a $\varepsilon_{o}>0$ such that

$$
u_{\varepsilon}(z) \leq a, \quad \forall z \in \Omega, \forall \varepsilon<\varepsilon_{o} .
$$

To conclude the proof observe that $u_{\varepsilon}$ becomes a solution of the subcritical case (1.8) with $h$ given by

$$
h(s)= \begin{cases}f(s)+s^{2^{*}-1}, & \text { if } s \leq a, \\ f(s)+\frac{\left(2^{*}-1\right)}{(\theta-1)} a^{2^{*}-\theta} s^{\theta-1}-\frac{\left(2^{*}-\theta\right)}{(\theta-1)} a^{2^{*}-1}, & \text { if } s>a,\end{cases}
$$

where $\theta>2$ is that one fixed in condition $\left(f_{4}\right)$. It is easy to check that $h$ is a $C^{1, \alpha}$ function, $h$ and $H(s)=\int_{o}^{s} h(\tau) d \tau$ satisfy $\left(\mathrm{f}_{1}\right),\left(\mathrm{f}_{2}\right),\left(\mathrm{f}_{3}\right),\left(\mathrm{f}_{4}\right)$, and $\left(\mathrm{f}_{5}\right)$. Let ${\tilde{J_{\varepsilon}}}_{\mathrm{\varepsilon}}$ be the $C^{1}$-functional on $H_{o}^{1}(\Omega)$ given by

$$
\tilde{J}_{\varepsilon}(u)=\frac{1}{2} \int_{\Omega}\left(\varepsilon^{2}|\nabla u|^{2}+u^{2}\right) d z-\int_{\Omega} H(u) d z .
$$

Since $f(s)+s^{2^{*}-1} \geq h(s)$ for all $s>0$, we have that

$$
J_{\varepsilon}(u) \leq \tilde{J}_{\varepsilon}(u), \quad \forall u \in H_{o}^{1}(\Omega),
$$

$J_{\varepsilon}\left(u_{\varepsilon}\right)=\tilde{J}_{\mathcal{\varepsilon}}\left(u_{\varepsilon}\right), J_{\varepsilon}^{\prime}\left(u_{\varepsilon}\right)=\tilde{J}_{\varepsilon}^{\prime}\left(u_{\varepsilon}\right)=0$. We conclude that $u_{\varepsilon}$ is a least-energy solution of the subcritical problem (1.8).

Lemma 2.8. (i) If $\tilde{c}_{\varepsilon}$ is the minimax level of $\tilde{J}_{\varepsilon}$, then $\tilde{c}_{\varepsilon}=c_{\varepsilon}$;

(ii) each $u_{\varepsilon}$ is a critical point of $\tilde{J}_{\varepsilon}$ in the minimax level and satisfies (1.8).

Since global problem (1.9) has a unique nondegenerate positive solution (cf. $[9,10]$ ), Theorem 1.1 comes from [8, Theorem 2.2] applied to the functional $\tilde{J}_{\varepsilon}$, and the asymptotic profile comes from [8, Theorem 2.3].

\section{Appendix}

Let $\Lambda$ be some general domain in $\mathbb{R}^{N}$ (bounded or unbounded). We will start with the following lemma due to Brézis and Kato [2].

Lemma A.1. Let $q \in L^{N / 2}(\Lambda)$ be a nonnegative function. Then, for every $\varepsilon>0$, there is a constant $\sigma_{\varepsilon}=\sigma(\varepsilon, q)>0$ such that

$$
\int_{\Lambda} q(x) u^{2} d x \leq \varepsilon \int_{\Lambda}|\nabla u|^{2} d x+\sigma_{\varepsilon} \int_{\Lambda} u^{2} d x, \quad \forall u \in H_{o}^{1}(\Lambda) .
$$


Remark A.2. If $q_{k} \rightarrow q$ in $L^{N / 2}(\Lambda)$, we can choose a constant $\sigma_{\varepsilon}$ independent of $k$. That is, $\sigma\left(\varepsilon, q_{k}\right)=\sigma_{\varepsilon}$ and

$$
\int_{\Lambda} q_{k}(x) u^{2} d x \leq \varepsilon \int_{\Lambda}|\nabla u|^{2} d x+\sigma_{\varepsilon} \int_{\Lambda} u^{2} d x, \quad \forall u \in H_{o}^{1}(\Lambda), k \in \mathbb{N} .
$$

Proof. Let $\sigma_{\varepsilon}=\sigma(\varepsilon, q)>0$ be such that

$$
\|q\|_{L^{N / 2}\left(\left\{q \geq \sigma_{\varepsilon}\right\}\right)} \leq \varepsilon S,
$$

where $S$ is a best constant in the Sobolev immersion $H_{o}^{1}(\Lambda) \hookrightarrow L^{2^{*}}(\Lambda)$, where $2^{*}=2 N /(N-2)$. For all $u \in H_{o}^{1}(\Lambda)$, we have

$$
\begin{aligned}
\int_{\Lambda} q(x) u^{2} d x & =\int_{\left\{q \geq \sigma_{\varepsilon}\right\}} q(x) u^{2} d x+\int_{\left\{q \leq \sigma_{\varepsilon}\right\}} q(x) u^{2} d x \\
& \leq \sigma_{\varepsilon} \int_{\left\{q \leq \sigma_{\varepsilon}\right\}} u^{2} d x+\int_{\left\{q \geq \sigma_{\varepsilon}\right\}} q(x) u^{2} d x \\
& \leq \sigma_{\varepsilon} \int_{\Lambda} u^{2} d x+\|q\|_{L^{N / 2}\left(\left\{q \geq \sigma_{\varepsilon}\right\}\right)}\|u\|_{L^{2 *}\left(\left\{q \geq \sigma_{\varepsilon}\right\}\right)}^{2}
\end{aligned}
$$

Inequality (A.1) follows from Sobolev estimate and the choice of $\sigma_{\varepsilon}$.

Remark 1.3 follows from the proof of Lemma A.1 and the inequality

$$
\int_{\Lambda} q_{k}(x) u^{2} d x \leq \int_{\Lambda} q(x) u^{2} d x+\left\|q_{k}-q\right\|_{L^{N / 2}(\Lambda)}\|u\|_{L^{2^{*}}(\Lambda)}^{2}
$$

Proof of Proposition 1.2. For any $n \in \mathbb{N}$ and $p>0$, consider $A_{n}=\left\{x \in \Lambda:|v|^{p} \leq\right.$ $n\}, B_{n}=\Lambda \backslash A_{n}$, and define $v_{n}$ by

$$
v_{n}=v|v|^{2 p} \quad \text { in } A_{n}, \quad v_{n}=n^{2} v \quad \text { in } B_{n} .
$$

Observe that $v_{n} \in H_{o}^{1}(\Lambda), v_{n} \leq|v|^{2 p+1}$ and

$$
\nabla v_{n}=(2 p+1)|v|^{2 p} \nabla v \quad \text { in } A_{n}, \quad \nabla v_{n}=n^{2} \nabla v \quad \text { in } B_{n} .
$$

So, using $v_{n}$ as a test function

$$
\int_{\Lambda} \nabla v \nabla v_{n} d x=\int_{\Lambda} g(x, v) v_{n} d x
$$

Using (A.7), we have

$$
\begin{aligned}
& (2 p+1) \int_{A_{n}}|v|^{2 p}|\nabla v|^{2} d x+n^{2} \int_{B_{n}}|\nabla v|^{2} d x \\
& \quad \leq \int_{\Lambda}\left|g(x, v) v_{n}\right| d x \leq \int_{\Lambda}\left(q(x)+C_{g}\right)\left|v v_{n}\right| d x .
\end{aligned}
$$


Now consider

$$
\omega_{n}=v|v|^{p} \quad \text { in } A_{n}, \quad \omega_{n}=n v \quad \text { in } B_{n} .
$$

Notice that $\omega_{n}^{2}=v v_{n} \leq|v|^{2(p+1)}$ and

$$
\nabla \omega_{n}=(p+1)|v|^{p} \nabla v \quad \text { in } A_{n}, \quad \nabla v_{n}=n \nabla v \quad \text { in } B_{n} .
$$

Therefore,

$$
\int_{\Lambda}\left|\nabla \omega_{n}\right|^{2} d x=(p+1)^{2} \int_{A_{n}}|v|^{2 p}|\nabla v|^{2} d x+n^{2} \int_{B_{n}}|\nabla v|^{2} d x .
$$

Combining (A.9) and (A.12), we obtain

$$
\frac{2 p+1}{(p+1)^{2}} \int_{\Lambda}\left|\nabla \omega_{n}\right|^{2} d x \leq \int_{\Lambda}\left(q(x)+C_{g}\right) \omega_{n}^{2} d x .
$$

Let $\sigma_{p}$ be given by Lemma A.1 with $\varepsilon=(2 p+1) / 2(p+1)^{2}$. Then

$$
\int_{\Lambda}\left|\nabla \omega_{n}\right|^{2} d x \leq \tilde{C}_{p} \int_{\Lambda} \omega_{n}^{2} d x
$$

where $\tilde{C}_{n}=\left(2(p+1)^{2} /(2 p+1)\right)\left(C_{g}+\sigma_{p}\right)$. Suppose that $v \in L^{2(p+1)}(\Lambda)$ for some $p \geq 2$. Applying Sobolev immersion in inequality (A.14) we have

$$
\left[\int_{A_{n}} \omega_{n}^{2^{*}} d x\right]^{2 / 2^{*}} \leq\left[\int_{\Lambda} \omega_{n}^{2^{*}} d x\right]^{2 / 2^{*}} \leq S \tilde{C}_{p} \int_{\Lambda}|v|^{2(p+1)} d x
$$

that is,

$$
\left[\int_{A_{n}}|v|^{2^{*}(p+1)} d x\right]^{2 / 2^{*}} d x \leq C_{p} \int_{\Lambda}|v|^{2(p+1)} d x
$$

where

$$
C_{p}=\frac{2(p+1)^{2}}{2 p+1} S\left(C_{g}+\sigma_{p}\right)
$$

Now, passing to the limit in (A.16) we have $v \in L^{2^{*}(p+1)}(\Lambda)$ and

$$
\|v\|_{L^{2^{*}(p+1)}(\Lambda)} \leq C_{p}\|v\|_{L^{2(p+1)}(\Lambda)} .
$$

The proof follows from the following iteration argument: let $p_{1}$ a positive such that $2\left(p_{1}+1\right)=2^{*}$. It is easy to see that $0<p_{1}$ and $v \in L^{2\left(p_{1}+1\right)}(\Lambda)$. Using inequality (A.18) we have

$$
v \in L^{2^{*}\left(p_{1}+1\right)}(\Lambda)
$$


Now choose $p_{2}$ such that $2\left(p_{2}+1\right)=2^{*}\left(p_{1}+1\right)$. It is easy to see that $0<$ $p_{1}<p_{2}$ and $v \in L^{p_{2}+1}(\Lambda)$. Using inequality (A.18) we have

$$
v \in L^{2^{*}\left(p_{2}+1\right)}(\Lambda)
$$

Continuing with this iteration we obtain an increasing sequence $p_{k}$ given by $2\left(p_{k+1}+1\right)=2^{*}\left(p_{k}+1\right)$ such that $v \in L^{2\left(p_{k+1}+1\right)}(\Lambda)$ for all $k \in \mathbb{N}$. From

$$
p_{k+1}+1=\frac{N}{N-2}\left(p_{k}+1\right)
$$

it follows that

$$
p_{k+1}+1=\left[\frac{N}{N-2}\right]^{k} 2^{*} .
$$

This shows that $p_{k}$ goes to $\infty$ and therefore,

$$
v \in L^{p}(\Lambda), \quad \forall p \geq 2
$$

Remark A.3. Proposition 1.2 is valid for positive subsolutions of problem (1.12) as we can check in its proof.

\section{Acknowledgments}

This work was done during the author's visit to the Department of Mathematics of Rutgers University. The author thanks Professor C. O. Alves for his suggestions improving the results. The author is supported by CNPq-Brazil.

\section{References}

[1] C. O. Alves, D. C. de Morais Filho, and M. A. S. Souto, Radially symmetric solutions for a class of critical exponent elliptic problems in $\mathbf{R}^{N}$, Electron. J. Differential Equations (1996), no. 07, 1-12.

[2] H. Brézis and T. Kato, Remarks on the Schrödinger operator with singular complex potentials, J. Math. Pures Appl. (9) 58 (1979), no. 2, 137-151.

[3] H. Brézis and L. Nirenberg, Positive solutions of nonlinear elliptic equations involving critical Sobolev exponents, Comm. Pure Appl. Math. 36 (1983), no. 4, 437-477.

[4] M. J. Esteban and P.-L. Lions, Existence and nonexistence results for semilinear elliptic problems in unbounded domains, Proc. Roy. Soc. Edinburgh Sect. A 93 (1982/83), no. 1-2, 1-14.

[5] D. Gilbarg and N. S. Trudinger, Elliptic Partial Differential Equations of Second Order, Springer-Verlag, New York, 1998.

[6] P.-L. Lions, The concentration-compactness principle in the calculus of variations. The locally compact case. II, Ann. Inst. H. Poincaré Anal. Non Linéaire 1 (1984), no. 4, 223-283.

[7] O. H. Miyagaki, On a class of semilinear elliptic problems in $\mathbf{R}^{N}$ with critical growth, Nonlinear Anal. 29 (1997), no. 7, 773-781. 
[8] W.-M. Ni and J. Wei, On the location and profile of spike-layer solutions to singularly perturbed semilinear Dirichlet problems, Comm. Pure Appl. Math. 48 (1995), no. 7, 731-768.

[9] M. A. S. Souto, Uniqueness of positive radial solutions of semilinear elliptic equations, preprint, 1999.

[10] Uniqueness of positive radial solutions of problem $-\Delta u+f(u)=0$, Anais do $49^{\circ}$ Seminário Brasileiro de Análise, Universidade Estadual de Campinas, Campinas, 1999, pp. 235-251.

[11] M. Struwe, Variational Methods. Applications to Nonlinear Partial Differential Equations and Hamiltonian Systems, Springer-Verlag, Berlin, 1990.

[12] G. Talenti, Best constant in Sobolev inequality, Ann. Mat. Pura Appl. (4) 110 (1976), 353-372.

[13] M. Willem, Minimax Theorems, Progress in Nonlinear Differential Equations and their Applications, vol. 24, Birkhäuser Boston, Massachusetts, 1996.

Marco A. S. Souto: Universidade Federal de Campina Grande, Departamento de Matemática e Estatística, Campina Grande-Pв, Cep 58109-970, Brazil

E-mail address: marco@dme.ufpb.br 


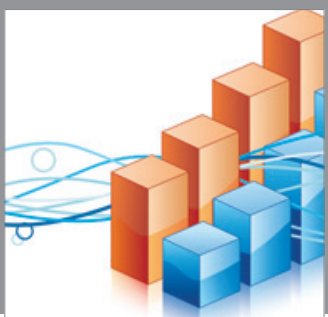

Advances in

Operations Research

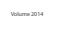

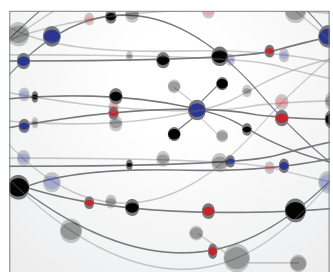

\section{The Scientific} World Journal
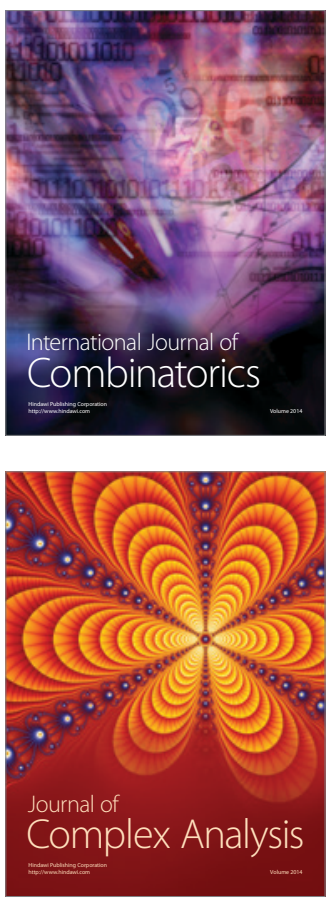

International Journal of

Mathematics and

Mathematical

Sciences
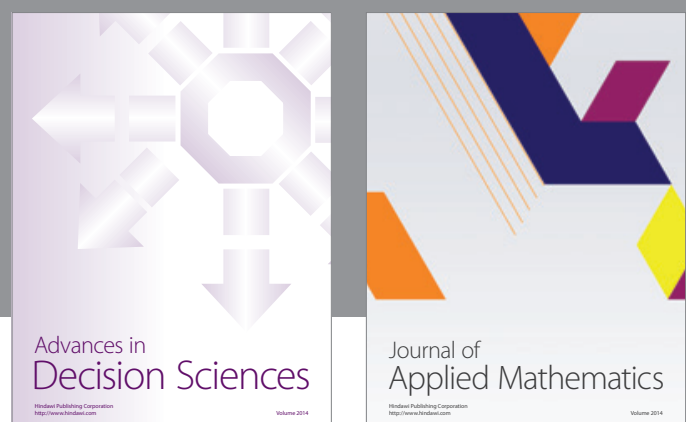

Journal of

Applied Mathematics
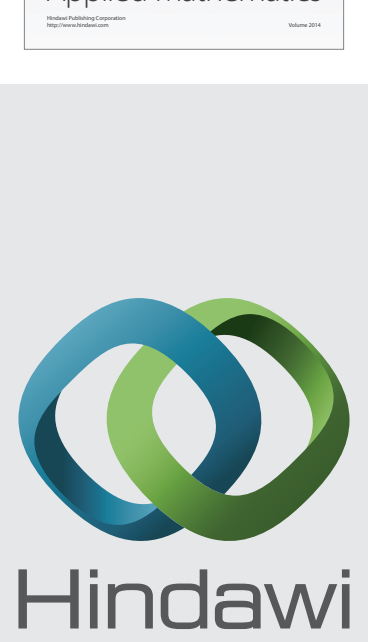

Submit your manuscripts at http://www.hindawi.com
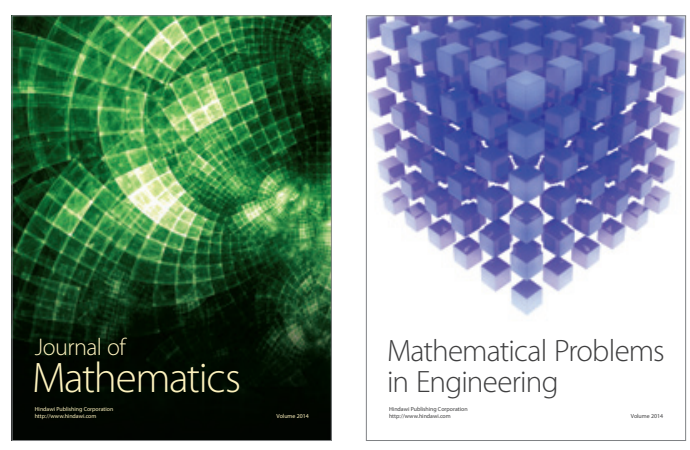

Mathematical Problems in Engineering
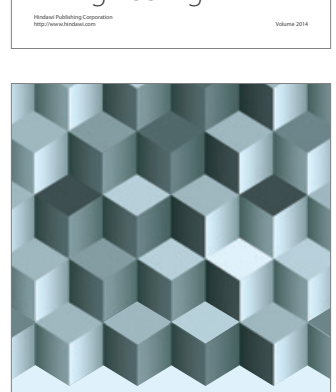

Journal of

Function Spaces
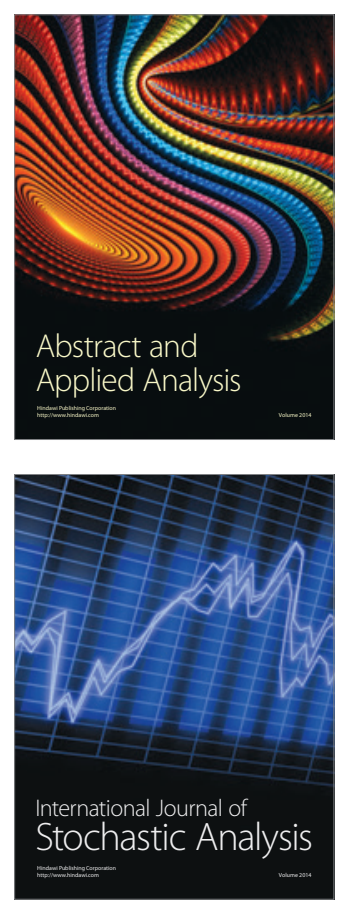

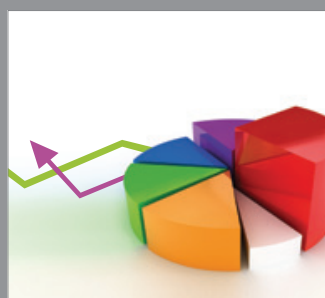

ournal of

Probability and Statistics

Promensencen
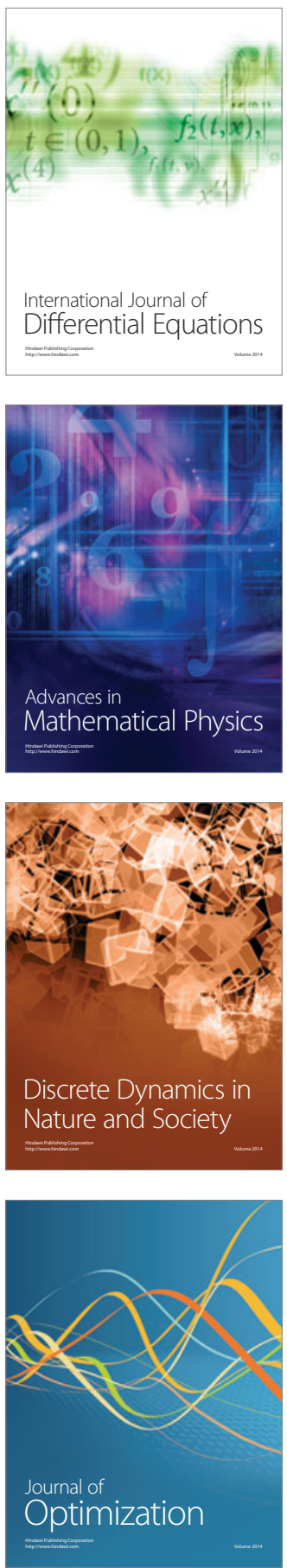